3 Francesca Moroni ${ }^{1}$ M.D., Benjamin Dwyer ${ }^{1}$ Ph.D., Catriona Graham ${ }^{2}$ M.Sc. , Chloe Pass ${ }^{3}$ 4 Ph.D., Laura Bailey ${ }^{3}$ Ph.D., Lisa Ritchie ${ }^{3}$, Donna Mitchell ${ }^{3}$, Alison Glover ${ }^{3}$, Audrey Laurie ${ }^{3}$, 5 Stuart Doig ${ }^{3}$, Emily Hargreaves ${ }^{3}$, Alasdair R. Fraser ${ }^{3}$ Ph.D., Marc L. Turner ${ }^{3}$ Ph.D., John D.M. 6 Campbell ${ }^{3}$ Ph.D., Neil W.A. McGowan ${ }^{3}$ Ph.D., Jacqueline Barry Ph.D. ${ }^{4}$, Joanna K. Moore ${ }^{1}$ 7

\section{Safety profile of autologous macrophage therapy for liver cirrhosis}

(1)

M.D., Peter C. Hayes ${ }^{5}$ Ph.D., Diana J. Leeming ${ }^{6}$ Ph.D., Mette J. Nielsen ${ }^{6}$ Ph.D., Kishwar Musa ${ }^{6}$

M.Sc., Jonathan A. Fallowfield ${ }^{5}$ Ph.D., Stuart J. Forbes ${ }^{\star 1}$ Ph.D.

${ }^{1} \mathrm{MRC}$ Centre for Regenerative Medicine, University of Edinburgh, UK; ${ }^{2}$ Edinburgh Clinical Research Facility, University of Edinburgh, UK; ${ }^{3}$ Tissues, Cells and Advanced Therapeutics, Scottish National Blood Transfusion Service (SNBTS), Edinburgh, UK; ${ }^{4}$ Cell and Gene Therapy Catapult, 12th Floor Tower Wing, Guy's Hospital, Great Maze Pond, London, UK; ${ }^{5}$ Centre for Inflammation Research, University of Edinburgh, UK; ${ }^{6}$ Nordic Biosience, Fibrosis Biology and Biomarkers, Herlev, Denmark.

\section{${ }^{*}$ Corresponding author:}

Professor Stuart J Forbes

Address: MRC Centre for Regenerative Medicine, University of Edinburgh, Edinburgh BioQuarter, 5 Little France Drive, Edinburgh, EH16 4UU, UK.

Telephone: $+44(0) 1316519510$

Email: stuart.forbes@ed.ac.uk 
Therapies to reduce liver fibrosis and stimulate organ regeneration are urgently needed. We conducted a first-in-human, phase 1 dose-escalation trial of autologous macrophage therapy in 9 adults with cirrhosis and Model for End-Stage Liver Disease (MELD) score of 10-16 (ISRCTN10368050). Groups of 3 participants received a single peripheral infusion of $10^{7}, 10^{8}$, or up to $10^{9}$ cells. Leukapheresis and macrophage infusion was well-tolerated with no transfusion reactions, dose-limiting toxicities or macrophage activation syndrome. All participants were alive and transplant-free at 1 year, with only 1 clinical event recorded, the occurrence of minimal ascites. The primary outcomes of safety and feasibility were met. This study informs and provides a rationale for efficacy studies in cirrhosis and other fibrotic diseases.

\section{BACKGROUND}

Globally, liver cirrhosis currently causes 1.16 million deaths every year. In the US, among people aged $45-64$ years, chronic liver disease is the $4^{\text {th }}$ leading cause of death. ${ }^{1}$ Causespecific interventions are effective, but patients often present with advanced liver disease and cirrhosis. No curative options are available for cirrhosis except for organ transplantation which requires major surgery and lifelong immunosuppression. Donor organ availability also restricts access to transplantation. ${ }^{2}$ Alternative therapies to treat cirrhosis are therefore being developed including cell therapies..$^{3,4}$

The macrophage is a cellular regulator of liver fibrosis deposition and resolution. ${ }^{5}$ During disease progression macrophages release signals which drive inflammatory cell recruitment and activation of hepatic stellate cells to produce extracellular matrix (ECM). Following cessation of injury, macrophages release matrix metalloproteinases (MMPs) that promote fibrotic ECM degradation, and factors that dampen the inflammatory response ${ }^{6-8,9}$ and drive 
In mouse models of liver fibrosis, macrophages injected via a peripheral vein home to the liver, express MMPs, and recruit host immune cells to liver scar via chemokine expression, ameliorating liver fibrosis, stimulating liver regeneration and improving function. ${ }^{10}$ Circulating $\mathrm{CD}_{14}{ }^{+}$monocytes can be isolated from cirrhotic patient mononuclear cell (MNC) leukapheresis products with high yield and purity and can be differentiated using Good Manufacturing Practice (GMP)-compliant processes into macrophages with a comparable phenotype to those from healthy volunteers. ${ }^{11,12}$ These macrophages can also resolve liver fibrosis in mouse models. ${ }^{12}$ These data prompted us to conduct a first-in-human, phase 1 , single-arm, dose-escalation clinical trial in people with cirrhosis evaluating maximum-tolerated dose and safety of peripheral infusion of ex vivo matured autologous monocyte-derived macrophages.

\section{RESULTS}

\section{Trial population, baseline and treatment characteristics}

11 participants ( 4 female and 7 male, mean age $58.54 \pm 5.85$ ) with compensated liver cirrhosis and MELD score between 10 and 16 attended a single centre (Royal Infirmary of Edinburgh, UK) for screening between 08 August 2016 and 27 March 2017 (Fig. 1). Two individuals did not meet screening criteria. Nine participants were enrolled in the trial and were followed-up for 1 year to 06 April 2018. Demographic and baseline characteristics of study participants are shown in Table 1. The mean duration of cirrhosis was $5.22 \pm 4.22$ years. All participants were abstinent from alcohol at the time of recruitment except for one individual who had a history of intermittent low-level alcohol consumption (1-10 units per week). A week before the planned treatment, participants underwent a standard leukapheresis to collect circulating monocytes. Monocytes were isolated from MNC and the Investigational Medical Product (IMP) produced in a GMP-accredited facility (Extended Data 1). 
Each group of 3 participants ( 9 in total) received a single infusion of autologous macrophages at $10^{7}, 10^{8}$ or up to $10^{9}$ cells, respectively in a dose-escalation manner. All participants were successfully evaluated for safety, feasibility and maximum-achieved safe dose of autologous macrophages. We also measured changes in: markers of liver fibrosis (serum Enhanced Liver Fibrosis (ELF ${ }^{\mathrm{TM}}$ ) test (Siemens Healthineers, UK), serum PRO-C3 and C3M (Nordic Bioscience, Denmark) and transient elastography (Fibroscan®, Echosens, France)); liver function (MELD and UKELD scores); health-related quality of life (HRQL) using the Chronic Liver Disease Questionnaire (CLDQ) instrument; transplant-free survival and number of clinical events related to decompensation of cirrhosis.

\section{Safety outcomes}

All participants completed 1-year of follow-up after macrophage infusion. No participants withdrew from the study and none developed acute transfusion reactions during macrophage infusion or in the $12 \mathrm{~h}$ post-infusion observation period. A total of 3 serious adverse events were recorded; these were assessed as mild in severity, unrelated to the IMP and there were no sequelae (Table 2). There were 70 adverse events documented in the reporting period (Table 2). A single clinical event occurred, described as a small volume of ascites around the liver on ultrasound. 9/22 (41\%), 8/19 (42\%) and 6/29 (21\%) adverse events were considered possibly related to the IMP in the $10^{7}, 10^{8}$ and up to $10^{9}$ cell dose groups, respectively. Overall, $56 \%$ of adverse events were considered unrelated to the IMP. No dose-toxicity relationships were identified. At the end of the study period all 9 participants were alive and transplant-free.

Serum ALT and bilirubin changes at 90 -days were respectively $0.88 \pm 0.21$ and $0.80 \pm 0.30$-fold from baseline. Fluctuation in platelet count is common in patients with cirrhosis and portal hypertension, but we did not observe a reduction in platelets to lower than $30 \%$ from baseline or clinically significant thrombocytopenia. The baseline total white cell count varied in this study population. As expected, total circulating leukocyte counts were affected by leukapheresis, but returned to baseline prior to infusion (7 days after leukapheresis). In some individuals we noted a small and transient increase in white cell count following infusion of 
macrophages which did not persist beyond 7 days post-infusion (Extended Data 2). Serum cytokines (including IL1 $\alpha$, IL6, IL8, IL10, TNF $\alpha$ and IFNy) did not change significantly from baseline (Extended Data 3). Specifically, levels of IL8 (which correlate with risk of macrophage activation syndrome (MAS)) decreased transiently after macrophage infusion, with a delta of $-8.23 \pm 14.39 \mathrm{pg} / \mathrm{mL}$ at 30 days and of $-1.58 \pm 13.54 \mathrm{pg} / \mathrm{mL}$ at 90 days.

\section{Secondary outcomes}

At day 90 following macrophage infusion, six out of 9 participants showed a decrease in MELD score (Fig. 2 and Extended Data 4). For all patients, the MELD at baseline was $11.88 \pm 1.40$ (range 9.90 to 13.87 ) with a mean $\Delta$-MELD at 90 days of $-1.12 \pm 1.87$ (range -4.90 to 1.76 ). (Fig. 2 and Extended data 4). At 1-year follow-up MELD decreased in 7 out of 9 participants; with a mean $\triangle$-MELD for all patients at 1 year of $-0.910 \pm 1.24$ (range -2.41 to 1.68 ). Overall, we did not observe a clear dose-related response; however, in the highest cell group the MELD scores all followed a similar downward trajectory over the period of follow up (Fig. 2). The mean $\Delta$-UKELD score for all participants at 90 days was $-0.42 \pm 2.27$. Serum albumin levels at 90 days showed little change from baseline in all participants with mean $\Delta$-albumin of $-0.20 \pm .0 .23 \mathrm{~g} / \mathrm{dL}$, with range +0.2 to -0.5 (Extended Data 5 ). Similarly, INR was unaffected in all participants by macrophage infusion, with mean \pm SD change from baseline of $-0.04 \pm 0.09$ and $-0.06 \pm 0.09$ at 90 days and 360 days respectively.

To detect a change in fibrosis, a range of non-invasive markers of liver fibrosis were quantified. The technical success rate of transient elastography was $91.66 \%$. Data not meeting the quality specification as per manufacturer recommendation were removed ( 2 baseline and 190 -day measurements). Baseline liver stiffness measurements were consistent with cirrhosis (mean $57.44 \pm 24.01 \mathrm{kPa}$ ). In 5 out of 9 participants liver stiffness measurements decreased by $>6$ $\mathrm{kPa}$ at 1 -year of follow-up, with an overall mean reduction of $-11.91 \pm 10.55 \mathrm{kPa}$ (Extended Data 6). While a change of $6 \mathrm{kPa}$ might be considered meaningful in the context of pre-cirrhotic 
liver fibrosis, ${ }^{13}$ the importance of this change in established cirrhosis is uncertain. There was a downward trend in ELF scores following macrophage infusion (Fig. 3a). The mean ELF score at baseline was $12.43 \pm 0.94$ with mean delta-ELF at 90 days of $-0.24 \pm 0.46$ and at 1 year of $1.13 \pm 1.21$ (Extended Data 7 ). There was a similar change in serological markers of type-III collagen turnover, with mean $\%$ change of $\mathrm{PRO}-\mathrm{C} 3$ of $-14.86 \pm 14.50$ and $\%$ change of $\mathrm{C} 3 \mathrm{M}$ of $-10.95 \pm 13.37 \mathrm{ng} / \mathrm{mL}$ at day 90 (Fig. 3b-c). The larger \% decrease in PRO-C3 could indicate a predominant decrease in fibrogenic activity following infusion of macrophages. Longitudinal of health-related quality of life scores (HRQL) assessment showed relatively small variations in composite Chronic Liver Disease Questionnaire (CLDQ) scores over time, but 5 out of 9 participants showed an improvement in overall HRQL at day 90 post-macrophage infusion (Fig. 3d and Extended Data 8). Individual domain scores are shown in Extended Data Table 1.

\section{DISCUSSION}

This first-in-human trial confirmed the safety and feasibility of a single peripheral infusion of autologous macrophages in participants with compensated liver cirrhosis of differing aetiology. Leukapheresis was well-tolerated by all participants with minimal side effects. Administration of macrophages was safe, with no clinically relevant adverse reactions recorded during the infusion or in the immediate post-infusion period. The $3+3$ trial dose-escalation model is designed to define a maximum-tolerated dose. Due to monocyte isolation and production limitations, we were able to generate a "maximum-achieved dose" of up to $10^{9}$ cells (specifically $0.8 \times 10^{9}$ cells), for which we sought to determine the safety and feasibility. As expected, in a study population with advanced cirrhosis and other co-morbidities, we observed adverse events throughout the study. One participant had a previous history of intermittent low-level alcohol consumption, but serial gamma-glutamyl transpeptidase (GGT) levels (a biochemical marker of alcohol consumption) remained static at all follow-up visits, suggesting that this did not influence the measured outcomes for this patient. Most of the 
adverse events recorded in the study were exacerbations of existing conditions or minor self-

158 limiting events. The 3 serious adverse events were considered mild and unrelated to the IMP. Among AEs possibly related to the IMP, none had Common Terminology Criteria for Adverse Events (CTCAE) severity grading over 2. There were no dose-related phenomena. All participants reached 360 days of follow-up and were transplant-free. We listed a single clinical event (worsening ascites) during the whole follow-up period. This was identified on ultrasound and resolved with diuretics. All other participants remained well compensated. Although we did not label the infused macrophages, previous animal models and human case reports ${ }^{14}$ suggest that macrophages infused via peripheral or central veins will transiently pass through the lungs, before engrafting in the liver and spleen. ${ }^{10,15,16}$ While this does not prove that the cell product used in our study reached the liver, these observations are supportive. We did not record any clinically meaningful changes in respiratory rate or oxygen saturation at any point during infusion or 12-hour follow-up period. Overall the IMP appeared safe during administration and the extended follow-up period of 360 days.

This single-arm phase 1 study was not designed or powered to demonstrate statistically significant changes in efficacy measures following macrophage therapy. However, in 6 of 9 participants reductions in MELD score were observed at 90 days, largely due to a decrease in serum bilirubin. This contrasts with a recent RCT using autologous CD133+ stem cells in adults with cirrhosis of comparable severity to this study which showed no improvement in MELD score. ${ }^{17}$ In one individual, total bilirubin and MELD score were higher at 360 days of follow-up compared to baseline; however, over $85 \%$ of the total bilirubin was unconjugated, cell infusion, including UKELD score and serum albumin. Overall, no robust dose-dependent treatment effects were observed in secondary outcomes. The macrophages manufactured using GMP-compliant processes have been comprehensively characterised and demonstrate a mature phenotype (CD14+ / high 25F9 
expression), plus retention of high levels of markers associated with tissue repair and inflammation resolution (CD206, CD163 and CD169). ${ }^{11}$

A number of non-invasive measures of liver fibrosis improved following macrophage infusion including transient elastography, serum ELF score and the collagen turnover markers PROC3 and C3M, highlighting the potential antifibrotic effect of autologous monocyte-derived macrophage infusion in cirrhosis.

There was variability in measured responses to macrophage infusion, even in participants treated with the same cell dose. This likely reflects the multiple factors that could determine the effect of macrophage infusion in an individual with cirrhosis such as duration and aetiology of liver disease, other comorbidities, or engraftment and survival of the infused macrophages in the liver. The influence of these variables will be better addressed in a larger randomised controlled phase 2 trial.

Impairment of HRQL is reported by most patients with advanced cirrhosis and HRQL scores improve significantly following liver transplantation. ${ }^{18}$ Given that a change of 0.5 on the 1 to 7 scale represents an important difference in CLDQ score, 5 of 9 participants exhibited an improvement in overall HRQL score at day 90 post-infusion. ${ }^{19}$ In the remaining participants, composite CLDQ scores were either unchanged $(n=2)$ or worse $(n=2)$ at 90 days. Interestingly, there was an improvement in most participants in the emotional domain at day 90 postinfusion. We noted an inverse association between delta-MELD and CLDQ scores. Moreover, in the 4 individuals in whom MELD failed to decrease or worsened, we observed no improvement in HRQL. ${ }^{19}$

This first-in-human study confirmed the safety, feasibility and maximum-achievable dose of autologous macrophages and facilitate future efficacy studies in cirrhosis and other fibrotic diseases. The effects of macrophage therapy upon efficacy measures including transplantfree survival, MELD and UKELD score, fibrosis markers and HRQL will be evaluated in an ongoing phase 2 randomised controlled trial (ISRCTN 10368050). 


\begin{tabular}{|c|c|c|c|c|c|c|c|c|c|c|c|}
\hline & \multicolumn{2}{|c|}{$\begin{array}{c}\text { Screen } \\
\text { Failure }(n=2)\end{array}$} & \multicolumn{3}{|c|}{$10^{7}$ Cells $(n=3)$} & \multicolumn{3}{|c|}{$10^{8}$ Cells $(n=3)$} & \multicolumn{3}{|c|}{ Up to $10^{9}$ Cells $(n=3)$} \\
\hline & & & & & & & & & $0.6 \times 10^{9}$ & $0.8 \times 10^{9}$ & $0.7 \times 10^{9}$ \\
\hline Participant ID & 001 & 002 & 003 & 004 & 005 & 006 & 007 & 008 & 009 & 010 & 011 \\
\hline \multicolumn{12}{|l|}{ DEMOGRAPHICS } \\
\hline Mean Age & \multicolumn{2}{|c|}{$63.00 \pm 5.66$} & \multicolumn{3}{|c|}{$59.33 \pm 8.50$} & \multicolumn{3}{|c|}{$55.67 \pm 6.35$} & \multicolumn{3}{|c|}{$57.67 \pm 2.88$} \\
\hline Body Mass Index & 32.1 & 28.2 & 24.7 & 29.6 & 35.6 & 26 & 27.8 & 27.8 & 33.6 & 27.6 & 29 \\
\hline Sex (Male:Female) & \multicolumn{2}{|c|}{$2: 0$} & \multicolumn{3}{|c|}{$1: 2$} & \multicolumn{3}{|c|}{$3: 0$} & \multicolumn{3}{|c|}{$1: 2$} \\
\hline Ethnicity & \multicolumn{2}{|c|}{ All Caucasian } & \multicolumn{3}{|c|}{ All Caucasian } & \multicolumn{3}{|c|}{ All Caucasian } & \multicolumn{3}{|c|}{ All Caucasian } \\
\hline
\end{tabular}
AETIOLOGY OF LIVER DISEASE

\begin{tabular}{l|l|}
$\operatorname{ALD}(\mathrm{n})$ & 1 \\
$\operatorname{NAFLD}(\mathrm{n})$ & 1 \\
$\operatorname{HCV}(\operatorname{SVR})(n)$ & 0 \\
$\operatorname{PBC}(\mathrm{n})$ & 0
\end{tabular}

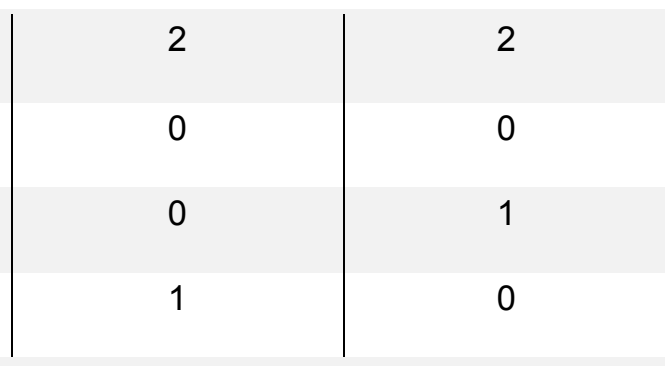

2

SEVERITY OF CIRRHOSIS

\begin{tabular}{|c|c|c|c|c|c|c|c|c|c|c|}
\hline MELD score & & 13 & 11 & 14 & 13 & 10 & 13 & 10 & 13 & 11 \\
\hline Mean MELD score & & \multicolumn{3}{|c|}{$12.37 \pm 1.51$} & \multicolumn{3}{|c|}{$11.90 \pm 1.48$} & \multicolumn{3}{|c|}{$11.36 \pm 1.62$} \\
\hline UKELD score & & 50 & 50 & 50 & 51 & 51 & 51 & 48 & 51 & 47 \\
\hline Child-Pugh score & & 6 & 5 & 7 & 6 & 6 & 8 & 5 & 9 & 9 \\
\hline Child-Pugh class & & A & A & B & A & A & B & A & B & B \\
\hline \multicolumn{11}{|c|}{ LIVER DISEASE COMPLICATIONS } \\
\hline Ascites & $x$ & $\mathrm{x}$ & & & & $x$ & $x$ & & $x$ & $x$ \\
\hline \multicolumn{11}{|l|}{ SBP } \\
\hline Variceal bleeding & & $x$ & & & & $x$ & $x$ & & $x$ & $x$ \\
\hline $\begin{array}{l}\text { Hepatic } \\
\text { encephalopathy }\end{array}$ & & & & & & & & & $\mathrm{x}$ & $\mathrm{x}$ \\
\hline
\end{tabular}

212 Table 1. Baseline characteristics of trial participants classified by cell dose group. ALD,

213 alcohol-related liver disease; NAFLD, non-alcoholic fatty liver disease; HCV, hepatitis C virus;

214 SVR, sustained viral response (> 6 months); PBC, primary biliary cholangitis; MELD, Model 
215 for End-Stage Liver Disease; UKELD, United Kingdom Model for End-Stage Liver Disease;

216 SBP, spontaneous bacterial peritonitis.

217

218

219

220

221

222

223

224

225

226

227

228

229

230

231

232

233

234 


\begin{tabular}{|c|c|c|c|}
\hline Adverse Event & $10^{7}$ cell dose & $10^{8}$ cell dose & $\begin{array}{l}\text { Up to } 10^{9} \text { cell } \\
\text { dose }\end{array}$ \\
\hline Nausea & 1 & 0 & 0 \\
\hline Abdominal pain & 0 & 2 & 3 \\
\hline Anorexia & 0 & 1 & 0 \\
\hline Light-headedness & 1 & 2 & 2 \\
\hline Fatigue & 1 & 1 & 3 \\
\hline Chest pain & 4 & 6 & 0 \\
\hline Joint pain/malaise & 2 & 2 & 3 \\
\hline Rash & 2 & 0 & 3 \\
\hline Hypocalcaemia symptoms (leukapheresis) & 1 & 2 & 3 \\
\hline Ascites & 0 & 1 & 0 \\
\hline Anaemia & 1 & 1 & 0 \\
\hline Infective & 3 & 0 & 2 \\
\hline Others & 5 & 1 & 10 \\
\hline TOTAL & 22 & 19 & 29 \\
\hline Number of probably related AEs & $9(41 \%)$ & $8(42 \%)$ & $6(21 \%)$ \\
\hline \multicolumn{4}{|l|}{ Type of Serious Adverse Event } \\
\hline Abdominal pain and constipation & & & 2 \\
\hline Papillary lesion of breast & 1 & & \\
\hline
\end{tabular}

Table 2. Recorded adverse events and serious adverse events during the study period.

237 Adverse events (AEs) and serious adverse events (SAEs) classified by dose, using Medical

238 Dictionary for Regulatory Activities (MedDRA) coding version 20.0. All AEs listed were defined as grade 1 or 2 according to the Common Terminology Criteria for Adverse Events version

240 5.0. All the SAE were considered unrelated to the macrophage infusion. Two, although rated 241 of mild severity, resulted in overnight admission to hospital. The SAE relative to the incidental 242 finding of a papillary lesion of breast through screening mammogram led to surgical excision 243 hence was considered moderate in severity. 
246 Fig. 1. Trial profile. A 3+3 model for dose escalation was used. During the study, there was 247 no dose-limiting toxicity (DLT); therefore, only 9 participants were needed to complete the 248 dose-escalation phase. 
265 Fig. 2. MELD score over time per cell dose group. Each line represents a participant in

266 the trial. Time-points indicate the time of macrophage infusion (purple line; approximately 14

267 days from baseline) and study-specific follow-up visits in the trial. Primary and secondary

268 outcomes were measured at day-90 post-infusion. a) $10^{7}$ cells; b) $10^{8}$ cells; c) $10^{9}$ cells. 
Fig. 3. Secondary outcomes a) Individual participant ELF score changes from baseline (BL) over time (delta-ELF). b) Individual participant PRO-C3 level changes from baseline over time (\% changes of PRO-C3). c) Individual participant C3M level changes from baseline over time (\% changes of C3M). d) Individual self-reported health related quality of life (HRQL) measures over time, expressed as the composite Chronic Liver Disease Questionnaire (CLDQ) score and not delta changes to highlight the significant variability in baseline HRQL composite score in this population. All data are shown by dose group $(n=3)$. 


\section{REFERENCES}

1. Asrani SK, Devarbhavi H, Eaton J, Kamath PS. Burden of liver diseases in the world. Journal of hepatology 2019;70:151-71.

2. D'Amico G, Garcia-Tsao G, Pagliaro L. Natural history and prognostic indicators of survival in cirrhosis: a systematic review of 118 studies. Journal of hepatology 2006;44:217-31.

3. Forbes SJ, Gupta S, Dhawan A. Cell therapy for liver disease: From liver transplantation to cell factory. J Hepatol 2015;62:S157-69.

4. Schuppan D, Kim YO. Evolving therapies for liver fibrosis. The Journal of clinical investigation 2013;123:1887-901.

5. Duffield JS, Forbes SJ, Constandinou CM, et al. Selective depletion of macrophages reveals distinct, opposing roles during liver injury and repair. J Clin Invest 2005;115:56-65.

6. Gouw AS, Clouston AD, Theise ND. Ductular reactions in human liver: diversity at the interface. Hepatology (Baltimore, Md) 2011;54:1853-63.

7. Boulter L, Govaere O, Bird TG, et al. Macrophage-derived Wnt opposes Notch signaling to specify hepatic progenitor cell fate in chronic liver disease. Nat Med 2012;18:572-9.

8. Ramachandran P, Pellicoro A, Vernon MA, et al. Differential Ly-6C expression identifies the recruited macrophage phenotype, which orchestrates the regression of murine liver fibrosis. Proc Natl Acad Sci U S A 2012;109:E3186-95.

9. Fallowfield JA, Mizuno M, Kendall TJ, et al. Scar-associated macrophages are a major source of hepatic matrix metalloproteinase-13 and facilitate the resolution of murine hepatic fibrosis. Journal of immunology (Baltimore, Md : 1950) 2007;178:5288-95.

10. Bird TG, Lu WY, Boulter $L$, et al. Bone marrow injection stimulates hepatic ductular reactions in the absence of injury via macrophage-mediated TWEAK signaling. Proc Natl Acad Sci U S A 2013;110:6542-7.

11. Fraser AR, Pass C, Burgoyne $P$, et al. Development, functional characterization and validation of methodology for GMP-compliant manufacture of phagocytic macrophages: A novel cellular therapeutic for liver cirrhosis. Cytotherapy 2017;19:1113-24.

12. Moore JK, Mackinnon AC, Wojtacha D, et al. Phenotypic and functional characterization of macrophages with therapeutic potential generated from human cirrhotic monocytes in a cohort study. Cytotherapy 2015;17:1604-16.

13. Foucher J, Chanteloup E, Vergniol J, et al. Diagnosis of cirrhosis by transient elastography (FibroScan): a prospective study. Gut 2006;55:403-8.

14. Hutchinson JA, Riquelme P, Sawitzki B, et al. Cutting Edge: Immunological consequences and trafficking of human regulatory macrophages administered to renal transplant recipients. Journal of immunology (Baltimore, Md : 1950) 2011;187:2072-8.

15. Sharkey J, Starkey Lewis PJ, Barrow M, et al. Functionalized superparamagnetic iron oxide nanoparticles provide highly efficient iron-labeling in macrophages for magnetic resonance-based detection in vivo. Cytotherapy 2017;19:555-69.

16. Thomas JA, Pope C, Wojtacha D, et al. Macrophage therapy for murine liver fibrosis recruits host effector cells improving fibrosis, regeneration, and function. Hepatology 2011;53:2003-15.

17. Newsome PN, Fox R, King AL, et al. Granulocyte colony-stimulating factor and autologous CD133positive stem-cell therapy in liver cirrhosis (REALISTIC): an open-label, randomised, controlled phase 2 trial. Lancet Gastroenterol Hepatol 2018;3:25-36.

18. Younossi ZM, McCormick M, Price LL, et al. Impact of liver transplantation on health-related quality of life. Liver transplantation : official publication of the American Association for the Study of Liver Diseases and the International Liver Transplantation Society 2000;6:779-83.

19. Younossi ZM, Guyatt G, Kiwi M, Boparai N, King D. Development of a disease specific questionnaire to measure health related quality of life in patients with chronic liver disease. Gut 1999;45:295-300.

20. Le Tourneau C, Lee JJ, Siu LL. Dose escalation methods in phase I cancer clinical trials. Journal of the National Cancer Institute 2009;101:708-20.

21. Kullak-Ublick GA, Andrade RJ, Merz M, et al. Drug-induced liver injury: recent advances in diagnosis and risk assessment. Gut 2017;66:1154-64. 
22. Mehta RL, Kellum JA, Shah SV, et al. Acute Kidney Injury Network: report of an initiative to improve outcomes in acute kidney injury. Crit Care 2007;11:R31.

23. Henter Jl, Horne A, Arico M, et al. HLH-2004: Diagnostic and therapeutic guidelines for hemophagocytic lymphohistiocytosis. Pediatr Blood Cancer 2007;48:124-31.

24. Tinegate $\mathrm{H}$, Birchall J, Gray A, et al. Guideline on the investigation and management of acute transfusion reactions. Prepared by the BCSH Blood Transfusion Task Force. British journal of haematology 2012;159:143-53.

25. Day J PP, Parkes J, et al. Derivation and Performance of Standardized Enhanced Liver Fibrosis (ELF) Test Thresholds for the Detection and Prognosis of Liver Fibrosis. . the journal of applied laboratory medicine 2018;3.

26. Friedrich-Rust M, Rosenberg W, Parkes J, Herrmann E, Zeuzem S, Sarrazin C. Comparison of ELF, FibroTest and FibroScan for the non-invasive assessment of liver fibrosis. BMC gastroenterology 2010;10:103. 27. Karsdal MA, Nielsen MJ, Sand JM, et al. Extracellular matrix remodeling: the common denominator in connective tissue diseases. Possibilities for evaluation and current understanding of the matrix as more than a passive architecture, but a key player in tissue failure. Assay and drug development technologies 2013;11:70-92.

28. Nielsen MJ, Nedergaard AF, Sun S, et al. The neo-epitope specific PRO-C3 ELISA measures true formation of type III collagen associated with liver and muscle parameters. American journal of translational research 2013;5:303-15.

29. Veidal SS, Vassiliadis E, Barascuk N, et al. Matrix metalloproteinase-9-mediated type III collagen degradation as a novel serological biochemical marker for liver fibrogenesis. Liver international : official journal of the International Association for the Study of the Liver 2010;30:1293-304.

30. Leeming DJ, Veidal SS, Karsdal MA, et al. Pro-C5, a marker of true type V collagen formation and fibrillation, correlates with portal hypertension in patients with alcoholic cirrhosis. Scand J Gastroenterol 2015;50:584-92.

31. Karsdal MA, Hjuler ST, Luo Y, et al. Assessment of liver fibrosis progression and regression by a serological collagen turnover profile. American journal of physiology Gastrointestinal and liver physiology 2019;316:G25-g31.

32. Huo $\mathrm{TI}, \mathrm{Wu} \mathrm{JC}$, Lin $\mathrm{HC}$, et al. Evaluation of the increase in model for end-stage liver disease (DeltaMELD) score over time as a prognostic predictor in patients with advanced cirrhosis: risk factor analysis and comparison with initial MELD and Child-Turcotte-Pugh score. Journal of hepatology 2005;42:826-32.

33. Barber K, Madden S, Allen J, Collett D, Neuberger J, Gimson A. Elective liver transplant list mortality: development of a United Kingdom end-stage liver disease score. Transplantation 2011;92:469-76.

34. Loria A, Escheik C, Gerber NL, Younossi ZM. Quality of life in cirrhosis. Current gastroenterology reports 2013;15:301.

\section{METHODS}

\section{Study oversight}

The MATCH 0.1 trial is an investigator-led study, funded by the Medical Research Council (Reference: MR/M007588/1) and sponsored by ACCORD (Academic and Clinical Central Office for 
Research and Development for NHS Lothian/University of Edinburgh). All study-related documents were designed by the trial team with input from ACCORD, an independent statistician and the Scottish National Blood Transfusion Service (SNBTS) team. The trial was approved by Scotland A Research Ethics Committee (Reference: 15/SS/0121), NHS Lothian Research and Development department and the Medicine and Health Care Regulatory Agency (MHRA-UK). The trial was registered in the International Standard Randomized Controlled Trial registry (ISRCTN10368050) and the European Clinical Trial Database (Reference: 2015-000963-15). All participants enrolled in the study gave informed consent and the trial was conducted under Good Clinical Practice regulations.

\section{Study design}

A phase 1 first-in-human trial using a standard $3+3$ dose-escalation design was conducted in a single centre (Royal Infirmary of Edinburgh, Edinburgh, UK). ${ }^{20}$ Due to limitations in production and cell selection, the maximum number of cells that could be produced for infusion was $10^{9}$; this study was therefore designed to ascertain the tolerability of the maximum-achievable dose and not the maximum-tolerated dose. This approach was approved by the appropriate oversight bodies (Phase I/First in Human Study Review Committee, Data Monitoring Committee and Trial Steering Committee). Escalation decisions were taken by an independent Data Monitoring Committee and recommendations discussed within the Trial Steering Committee and acted upon before each doseescalation.

\section{Study participants}

All participants were recruited through the hospital outpatient service in NHS Lothian between 08 August 2016 and 06 April 2018. 9 adult participants with liver cirrhosis of different aetiologies and a MELD score between 10 and 16 were enrolled. To confirm eligibility only, we used a MELD calculator adopted by the transplant coordinators within our unit; this rounds MELD score to the nearest integer. Full inclusion and exclusion criteria are detailed in the protocol in the Extended Data. Inclusion criteria included: age 18-75; MELD score 10-16 (inclusive); liver disease aetiology of alcohol-related liver disease, primary biliary cholangitis, non-alcoholic fatty liver disease, cryptogenic cirrhosis, haemochromotosis, alpha-1 antitrypsin deficiency or treated chronic hepatitis C (sustained viral 
response); liver cirrhosis (diagnosed by at least one of: liver biopsy, Fibroscan ${ }^{\mathrm{TM}}$ median liver stiffness measurement $>15 \mathrm{kPa}$, or clinical and radiological evidence consistent with cirrhosis). Exclusion criteria included: history of decompensated cirrhosis in the previous 3 months (portal hypertensive bleeding, ascites requiring medical treatment or hepatic encephalopathy requiring hospitalisation); hepatocellular carcinoma or undetermined liver nodules; cancer in the previous 5 years (excluding adequately treated and localised skin cancer or carcinoma-in-situ of the cervix); previous organ or tissue transplantation; listed for liver transplant; pregnancy and breastfeeding; presence of acute illness that may compromise safety of the patient in the trial. No active alcohol misuse $\geq 6$ calendar months prior to screening was permitted. Individuals attended for a screening visit to ensure eligibility $7 \pm 4$ days before scheduled leukapheresis. Participants underwent leukapheresis a week before infusions. The Investigational Medical Product (IMP) was produced in a GMP-accredited facility. On the day of infusion, active infection was excluded by physical examination and laboratory investigations. Prior to infusion, $10 \mathrm{mg}$ i.v. chlorphenamine and $100 \mathrm{mg}$ i.v. hydrocortisone was administered. Each group of 3 participants received a single infusion given over $30+/-5$ minutes of $10^{7}, 10^{8}$ and up to $10^{9}$ cells, respectively.

\section{Study Assessments}

During infusion, participants were monitored closely and observed overnight in the RIE Clinical Research Facility (CRF). Special arrangements were in place with the intensive care unit in the event of a severe reaction. The following morning full blood count, renal function, electrolytes, liver function tests, triglycerides and ferritin were checked prior to discharge to exclude toxicity, including Macrophage Activation Syndrome (MAS).

During the first two follow-up visits (day 7 and day 14 after IMP infusion) safety, dose-limiting toxicity (DLT) and the presence of MAS were assessed. The definition of DLT was formulated using accepted criteria ${ }^{21-24}$. serum creatinine $\geq 1.5$-fold from baseline, haemoglobin 1.5 -fold $\leq$ baseline, platelets $<2$-fold from baseline, total white cell count $<2.0 \times 10^{9}$, alanine aminotransferase $(\mathrm{ALT})>$ 3-fold from baseline, total bilirubin $>$ 3-fold from baseline, MELD score $>4$ points from baseline. Thereafter, participants were followed up at day 30,60, 90, 180 and 360 after IMP infusion with 
routine and biomarker blood tests, abdominal ultrasound, transient elastography and health-related quality of life (HRQL) assessment (full details are provided in the Protocol in the Extended Data). Transient elastography (Fibroscan ${ }^{\circledR}$, Echosens, France) is a well-validated non-invasive test to quantify liver fibrosis. It records the velocity of a sound wave passing through the liver and then converts that measurement into a liver stiffness value (expressed in kilopascals $(\mathrm{kPa})$ ). ${ }^{13}$

A range of serological biomarker tests are available for assessment of liver fibrosis. We used the Enhanced Liver Fibrosis (ELF ${ }^{\mathrm{TM}}$ test (Siemens Healthineers, UK)), a biochemical panel comprising serum markers that are indicators of ECM metabolism (hyaluronic acid, procollagen-III N-terminal pro-peptide (PIIINP), and tissue inhibitor of matrix metalloproteinase-1 (TIMP-1)). The composite ELF score has been validated for detection of liver fibrosis and for prognostication in chronic liver disease. ${ }^{25,26}$ By serological assessment of specific ECM fragments it may be possible to separate tissue formation from tissue degradation. ${ }^{27}$ We also measured PRO-C3 and C3M (Nordic Bioscience Protein Fingerprint ${ }^{\mathrm{TM}}$ technology) which are two markers derived from type-III collagen remodeling, i.e. N-terminal pro-peptide and MMP-9 degraded collagen fragment from the helix region, respectively, ${ }^{28,29}$ with utility for staging liver fibrosis and monitoring response to antifibrotic therapy ${ }^{30,31}$.

Liver function was assessed by the MELD and the United Kingdom Model for End-Stage Liver Disease (UKELD). These are established clinical scores calculated from objective variables (serum bilirubin, creatinine, International Normalized Ratio (INR) and sodium) that are used to estimate the severity of liver disease, determine prognosis and prioritize patients for transplantation. ${ }^{32,33}$

The Chronic Liver Disease Questionnaire (CLDQ) is a 29-item self-reported disease-specific instrument, measuring HRQL in the following domains: fatigue, activity, emotional function, abdominal symptoms, systemic symptoms, and worry. A composite score is calculated by the patient's response options in each domain using seven-point scales, ranging from the worst (1) to the best (7) possible function. The CLDQ is reliable, responsive and correlates with the severity of liver disease. ${ }^{19,34}$

Serum cytokines were analysed using a V-PLEX Human Biomarker 54-Plex kit on a MESO Quickplex SQ 120 according to the manufacturers' instructions (Meso Scale Discovery). We selected 
a set of 6 safety-related cytokines associated with 'cytokine storm' in MAS. These were IL8 (pivotal in the pathogenesis of MAS), IL1 $\alpha$, IL6, TNF $\alpha$, IFNY and IL10.

\section{Method of cell production}

The monocyte-derived macrophages were manufactured as previously described. ${ }^{11}$ Briefly, steadystate leukapheresis was collected from each patient (standard MNC program, 2.5 blood volume). Monocytes were isolated using a CliniMACS Prodigy ${ }^{\circledR}$ cell processor, programme LP14, tubing set TS510 with CliniMACS CD14 Reagent (all Miltenyi). Up to $3.5 \times 10^{10}$ TNC containing $4 \times 10^{9}$ CD14+ cells were processed in a single operation. Mean CD14+ cell purity was $98.3 \% \pm 0.7 \%$ and the mean selection yield of $55.25 \% \pm 5.4 \%$. A total of $2 \times 10^{9} \mathrm{CD} 14+$ cells were cultured in $4 \mathrm{x}$ gas-permeable plastic bags (MACS GMP cell differentiation bag 500 , Miltenyi Biotec) at $1 \times 10^{6}$ cells per $\mathrm{ml}$ in TexMACS GMP (phenol red-free) medium supplemented with $100 \mathrm{ng} / \mathrm{mL}$ M-CSF (GMP-grade, RND systems). Medium was replenished by removing $50 \%$ spent medium and replacing with $50 \%$ fresh medium supplemented with 200ng/mL M-CSF after 48 and 96 hours of culture. After 7 days, macrophages were harvested, counted and formulated into saline for injection supplemented to $0.5 \%$ Alburex (CSL Behring UK). Macrophages were characterized as viable, CD45+, CD14+, 25F9+ cells as previously described. ${ }^{11} \mathrm{CD} 14^{+}$monocytes were successfully isolated from all participants. A macrophage product was successfully manufactured and administered for all participants.

\section{Statistics}

A descriptive analysis of the primary outcome of safety and tolerability is presented. Secondary outcomes are presented graphically by dose and as changes from baseline. Unless stated, numerical data is expressed as mean \pm standard deviation (SD). A safety report was produced to review the day 14 results of the first participant, thereafter DMC reports were produced following the day 14 safety blood samples of each escalation group of 3 participants at each dose level or as required by serious adverse events. Any additional analysis was performed the end of the trial once the electronic database was locked following quality checks (QC). There was $100 \%$ QC of the data collected, with no missing data other than a single collagen biomarker sample at day 60 postinfusion. We report all adverse events by dose. 


\section{Data availability}

Data in the published article (and its Supplementary Information files) has been presented where possible in aggregated form. Any data presented to illustrate individual patient performance has been de-identified and only includes analysis of performance within the trial (such as MELD score). The datasets generated during and/or analysed during the current study are available from the corresponding author (SJF) upon reasonable request, although restrictions may apply due to patient privacy and General Data Protection Regulation.

\section{Acknowledgements}

This work was supported by a Medical Research Council UK grant (Biomedical Catalyst Major Awards Committee, Reference: MR/M007588/1) to Prof. S.J. Forbes.

We thank Prof. Zobair M. Younossi (Center for Outcomes Research in Liver Diseases, Washington DC, USA) for academic use of the CLDQ instrument and Prof. Lesley J. Fallowfield, (Sussex Health Outcomes Research \& Education in Cancer (SHORE-C), University of Sussex, UK) for advice about health-related quality of life assessment.

\section{Author Contributions}

Conceptualization and design of the work were carried out by SJF, CP, LR, LB, DM, AL, SD, EH, ARF, MLT, JDMC, NWAM, JB, JKM, PCH, JAF; the acquisition, analysis, and interpretation of data were performed by SJF, JAF, FM, BD, CG, DJL, MJN, KM; trial delivery and administration were carried out by FM, AG; the original draft of the manuscript was written by FM; the draft was reviewed and edited by all the authors.

\section{Competing interests}

JAF reports personal fees from Novartis, Ferring Pharmaceuticals, Galecto Biotech, Caldan Therapeutics, Gilde Healthcare, Arix Bioscience, Guidepoint and grants from GlaxoSmithKline, Novartis and Intercept Pharmaceuticals, outside the submitted work. SJF has a grant from Syncona 
to develop macrophages as a therapy. DJL, KM, MJN are full-time employees at Nordic Bioscience. DJL, MK and MJN are among the original inventors and patent holders of C3M and PRO-C3. DJL holds stock in Nordic Bioscience. PCH is an advisor for AbbVie, BMS, Eisai Ltd, Falk, Ferring, Gilead, Gore, Janssen, Lundbeck, MSD, Norgine, Novartis, ONO Pharmaceuticals, Pfizer and Roche, outside the submitted work. 